Supporting Information

\title{
Clobetasol propionate is a heme-mediated selective inhibitor of human cytochrome P450 3A5
}

William C. Wright ${ }^{1,2}$, Jude Chenge ${ }^{1}$, Jingheng Wang ${ }^{1}$, Hazel M. Girvan ${ }^{3}$, Lei Yang ${ }^{1}$, Sergio C. Chai $^{1}$, Andrew D. Huber ${ }^{1}$, Jing Wu ${ }^{1}$, Peter O. Oladimeji ${ }^{1}$, Andrew W. Munro ${ }^{3} \&$ Taosheng Chen $^{1 *}$

${ }^{1}$ Department of Chemical Biology and Therapeutics, St. Jude Children's Research Hospital, Memphis, Tennessee 38105-3678, USA.

${ }^{2}$ Integrated Biomedical Sciences Program, University of Tennessee Health Science Center, Memphis, Tennessee 38163, USA.

${ }^{3}$ Manchester Institute of Biotechnology, School of Natural Sciences, Department of Chemistry, The University of Manchester, Manchester, M1 7DN, UK.

\section{Contents:}

1) Supplementary Figures 1-7 (S2-S9)

2) Supplementary Data 1 and 2 (S10)

3) Supplementary Videos 1 and 2 (S11) 


\section{Supplementary Figures}
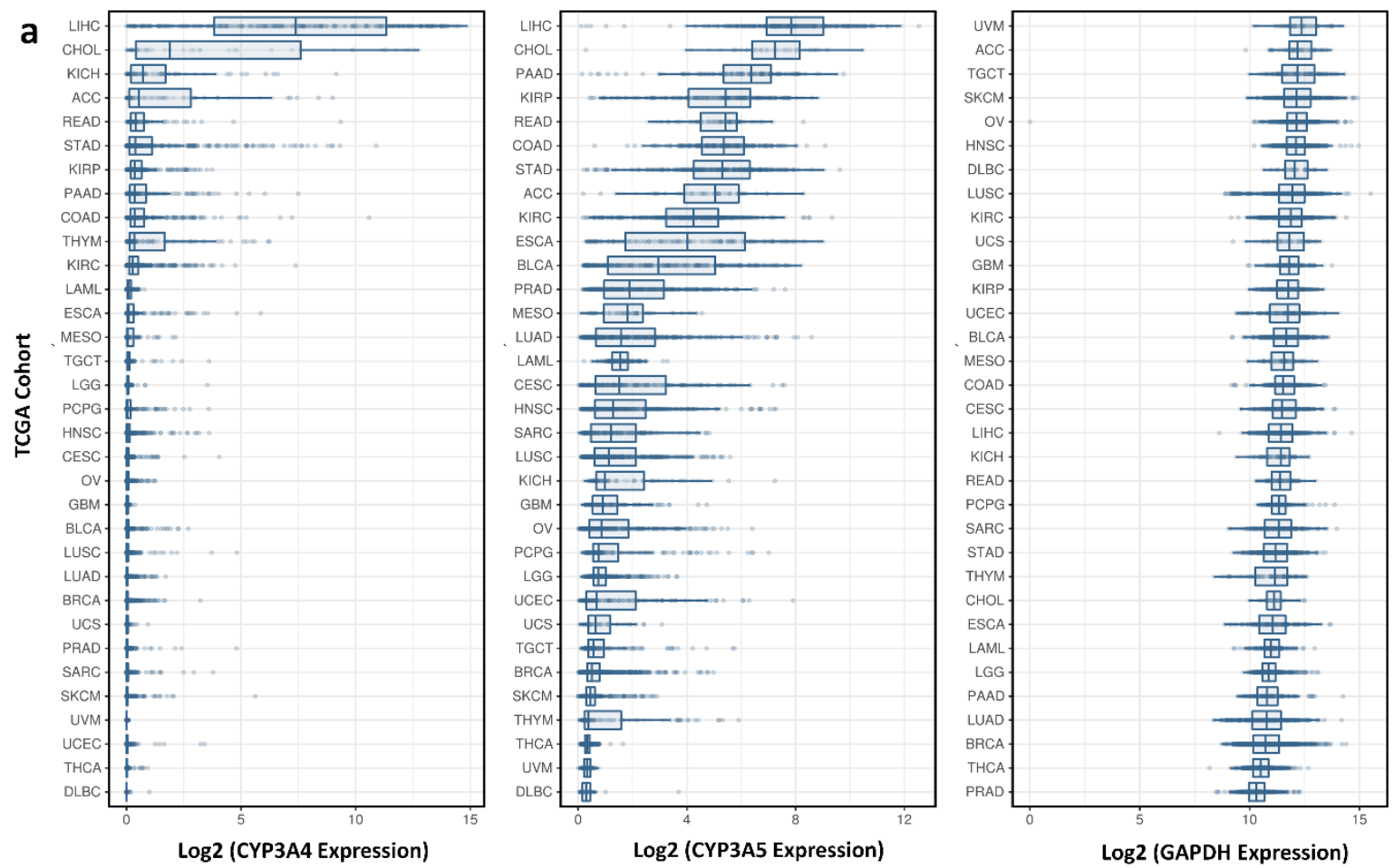

b

TCGA PanCancer Cohorts

ACC : Adrenocortical carcinoma

BLCA : Bladder Urothelial Carcinoma

LUAD : Lung adenocarcinoma

LUSC : Lung squamous cell carcinoma

MESO : Mesothelioma

BRCA : Breast invasive carcinoma

OV : Ovarian serous cystadenocarcinoma

CESC : Cervical squamous carcinoma \& endocervical adenocarcinoma $\mathrm{CHOL}$ : Cholangio carcinoma

COAD : Colon adenocarcinoma

DLBC : Lymphoid Neoplasm Diffuse Large B-cell Lymphoma

PAAD : Pancreatic adenocarcinoma

PCPG : Pheochromocytoma \& Paraganglioma

PRAD : Prostate adenocarcinoma

ESCA : Esophageal carcinoma

GBM : Glioblastoma multiforme

HNSC : Head and Neck squamous cell carcinoma

$\mathrm{KICH}$ : Kidney Chromophobe

KIRC : Kidney renal clear cell carcinoma

KIRP : Kidney renal papillary cell carcinoma

LAML : Acute Myeloid Leukemia

LGG : Brain Lower Grade Glioma

LIHC : Liver hepatocellular carcinoma

READ : Rectum adenocarcinoma

SARC : Sarcoma

SKCM : Skin Cutaneous Melanoma

STAD : Stomach adenocarcinoma

TGCT : Testicular Germ Cell Tumors

THCA : Thyroid carcinoma

THYM : Thymoma

UCEC : Uterine Corpus Endometrial Carcinoma

UCS : Uterine Carcinosarcoma

UVM : Uveal Melanoma

\section{Supplementary Figure 1. Normalized expression of CYP3A4 and CYP3A5 across various}

cancer types. (a) CYP3A4 (left) and CYP3A5 (middle) expression across all TCGA PanCancer

cohort types, with GAPDH (right) shown as a housekeeping example. All data were normalized

according to our RNA-seq reprocessing protocol (available in the protocol exchange: DOI:

10.21203/rs.2.16081/v1). (b) TCGA PanCancer cohort abbreviations. 

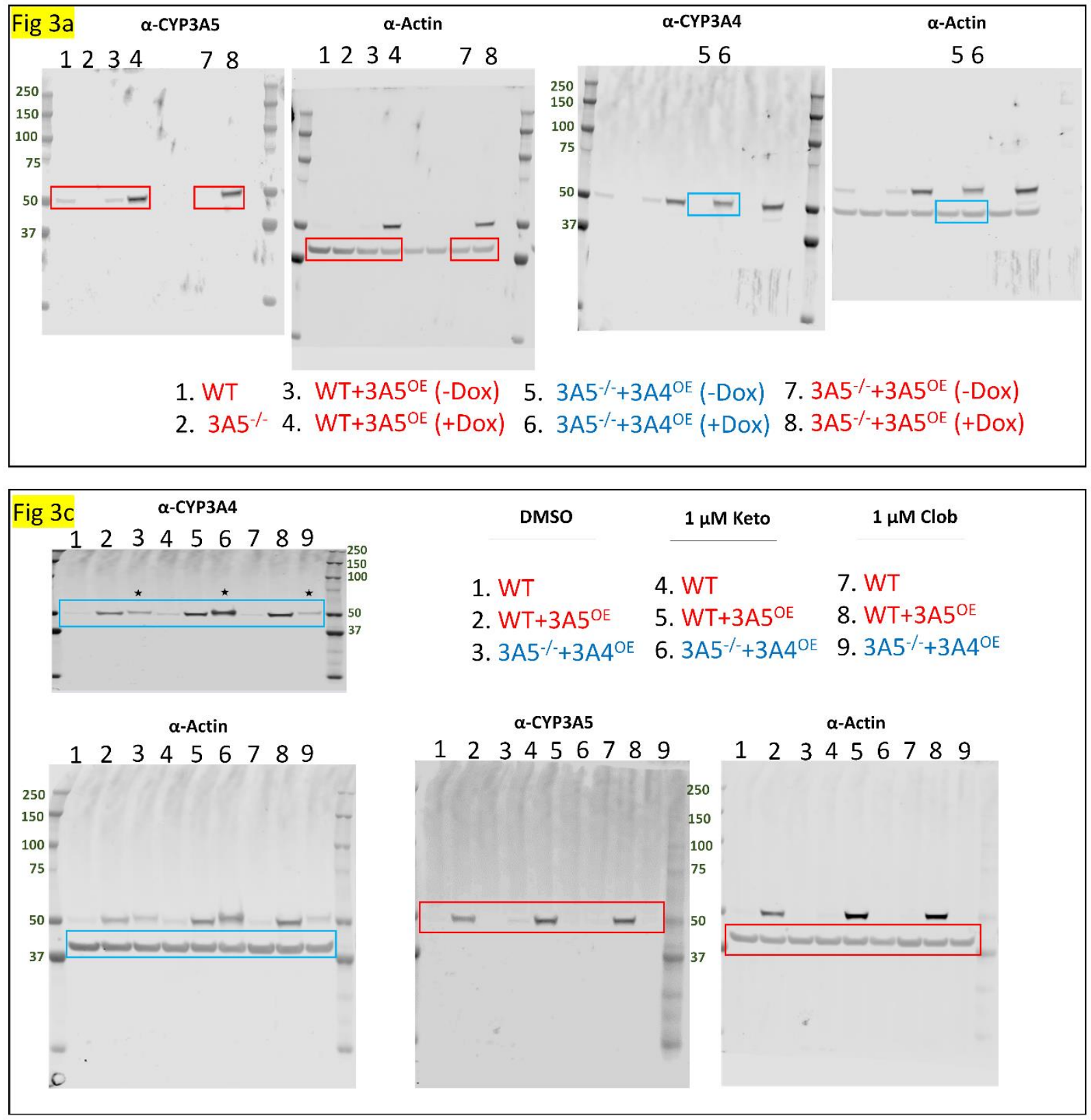

Supplementary Figure 2. Full-gel images of representative Western blots displayed in

Figure 3a (upper) and Figure 3c (lower). 


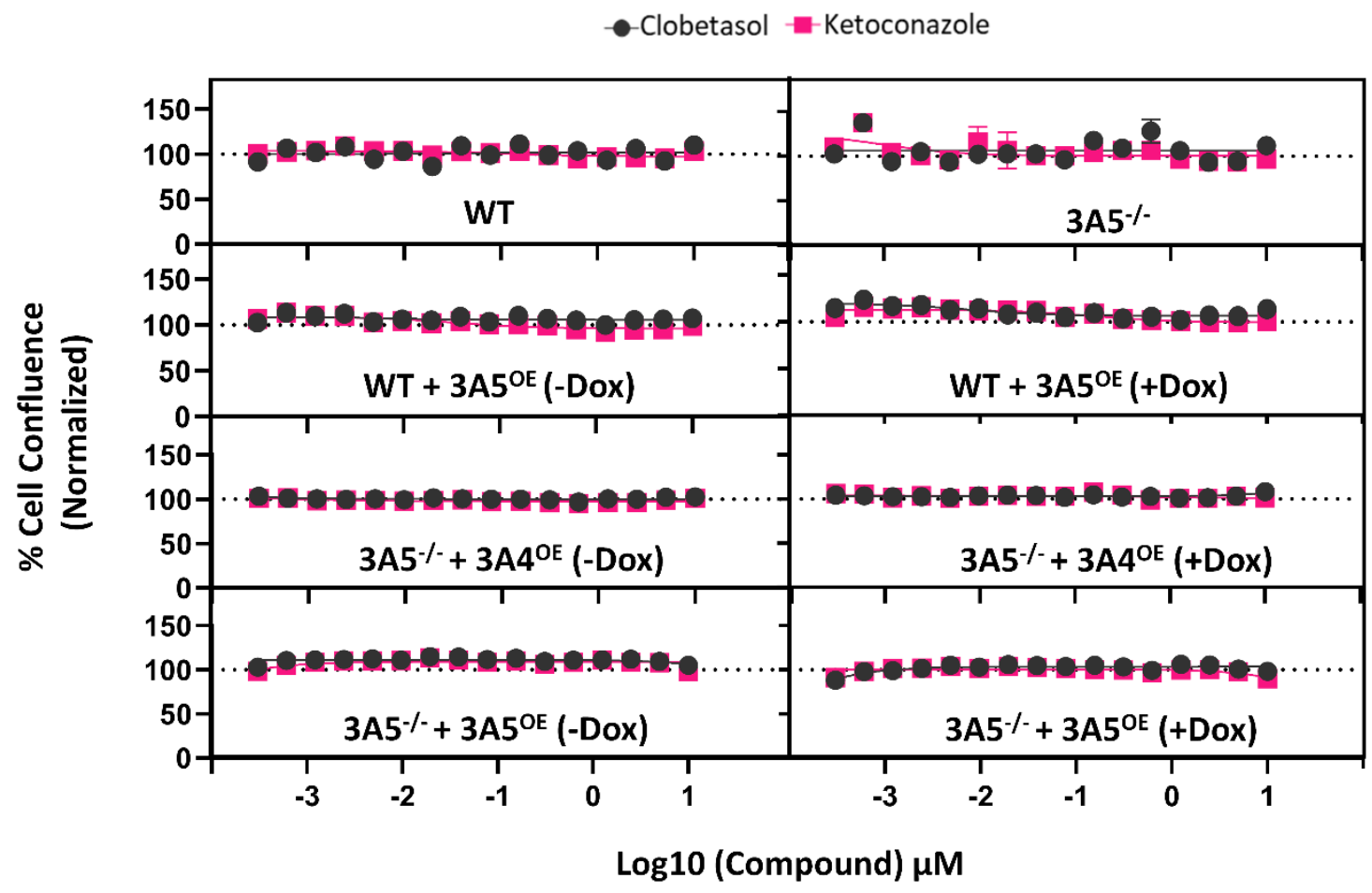

Supplementary Figure 3. Effect of clobetasol and ketoconazole on cell growth. Cell confluence measurements for various cell lines when treated with multiple concentrations of clobetasol (dark gray) or ketoconazole (pink). Confluence was normalized to DMSO for 100\% confluence. 

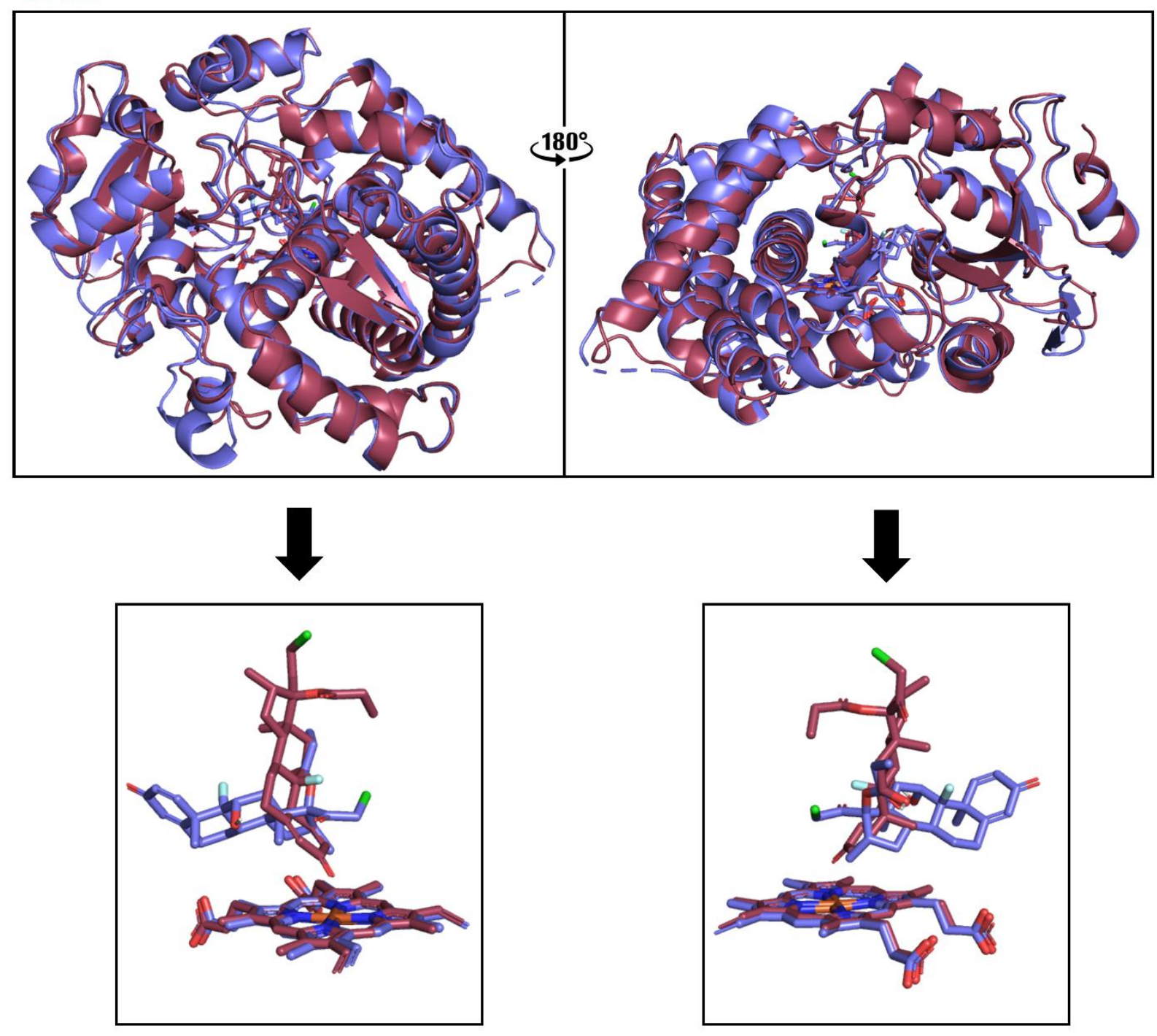

Supplementary Figure 4. Overlay of clobetasol docked into CYP3A4 and CYP3A5. Overlay of secondary structures for CYP3A4 (blue) and CYP3A5 (raspberry) illustrating the similarity of the structures (upper panels) and the differing binding orientations of clobetasol (lower panels). 


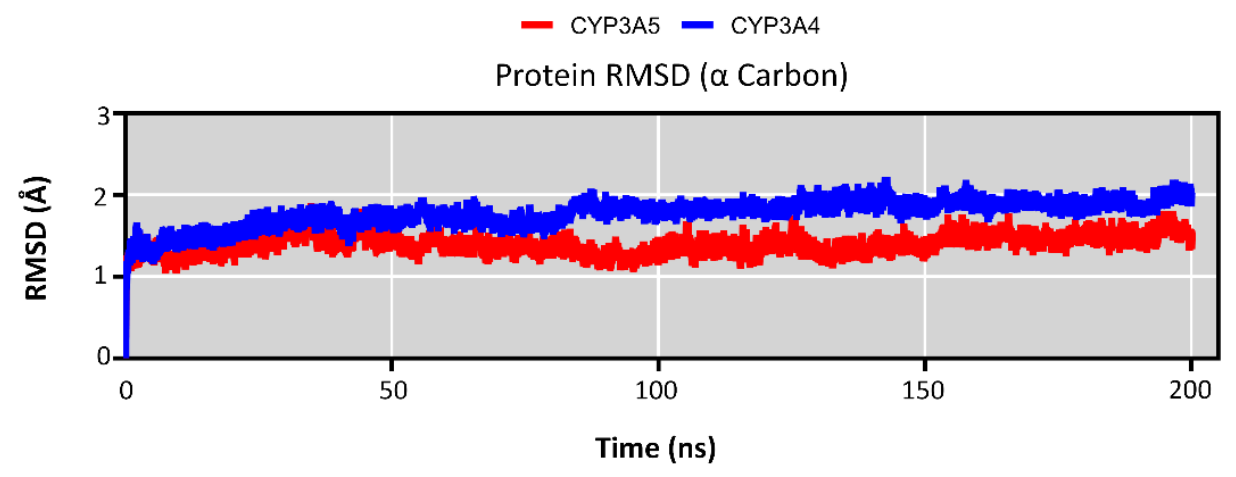

Supplementary Figure 5. RMSD of CYP3A4 and CYP3A5 molecular dynamics

simulations. Root mean square deviation (RMSD) in Ångstroms of the CYP3A4 (blue) and CYP3A5 (red) molecular dynamics simulations. RMSD was measured based on $\alpha$-carbon atoms. 
a
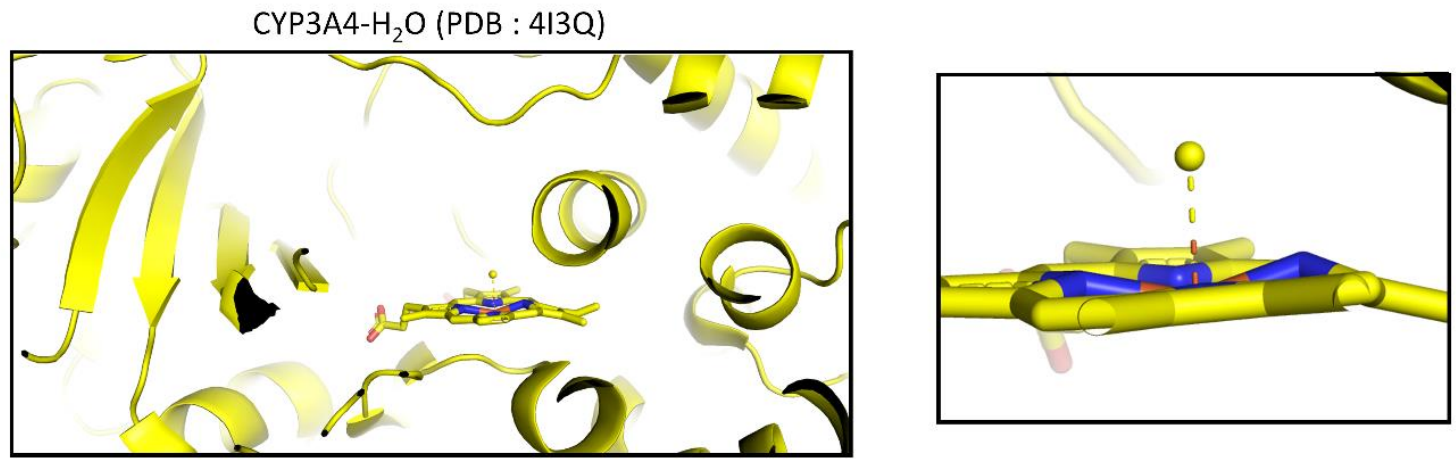

b
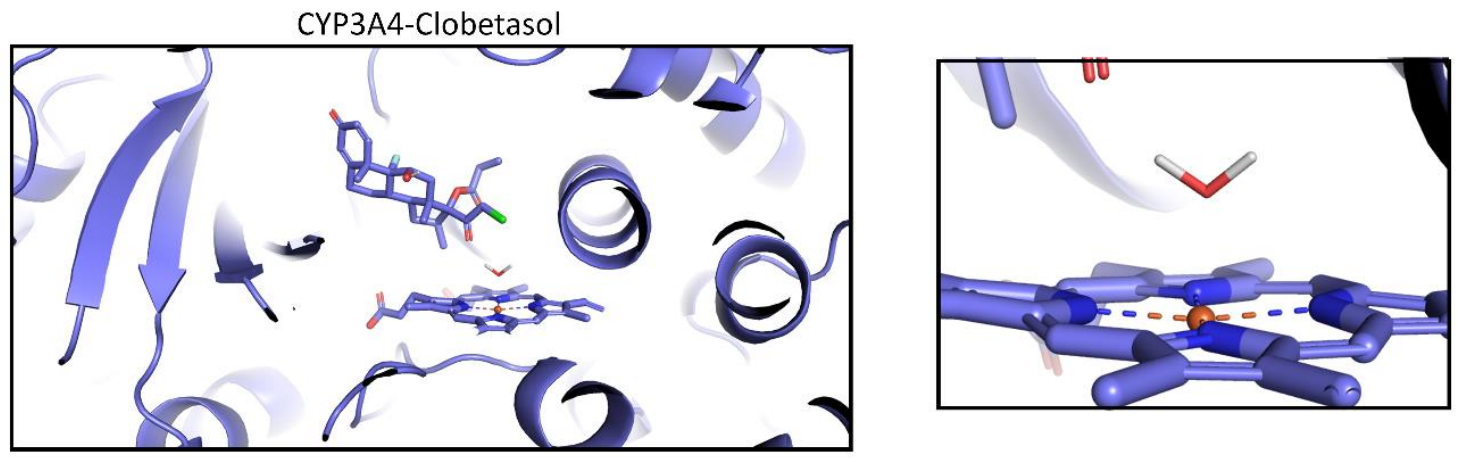

C
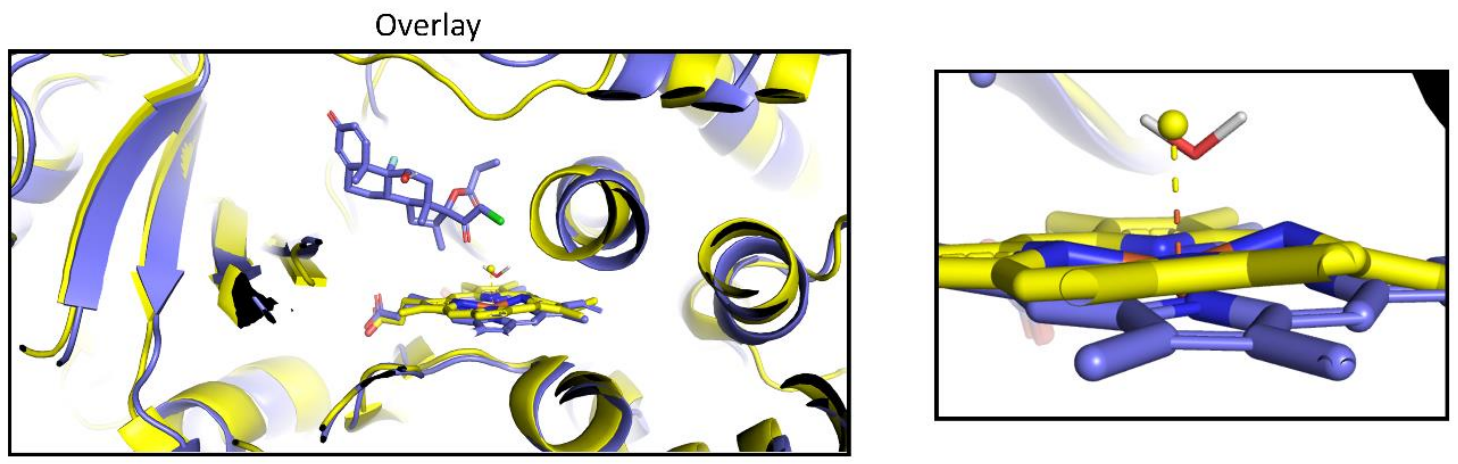

Supplementary Figure 6. Overlay of crystalized and simulated water-bound CYP3A4. (a)

CYP3A4 bound to a water molecule from a published crystal structure (PDB: 4I3Q). (b) The molecular dynamics simulation of CYP3A4 with clobetasol. (c) The two structures superimposed. 
a

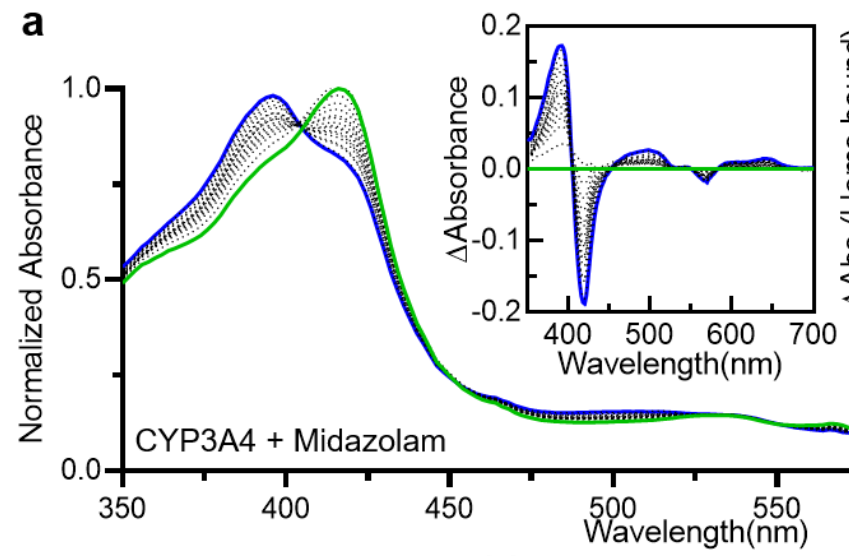

b
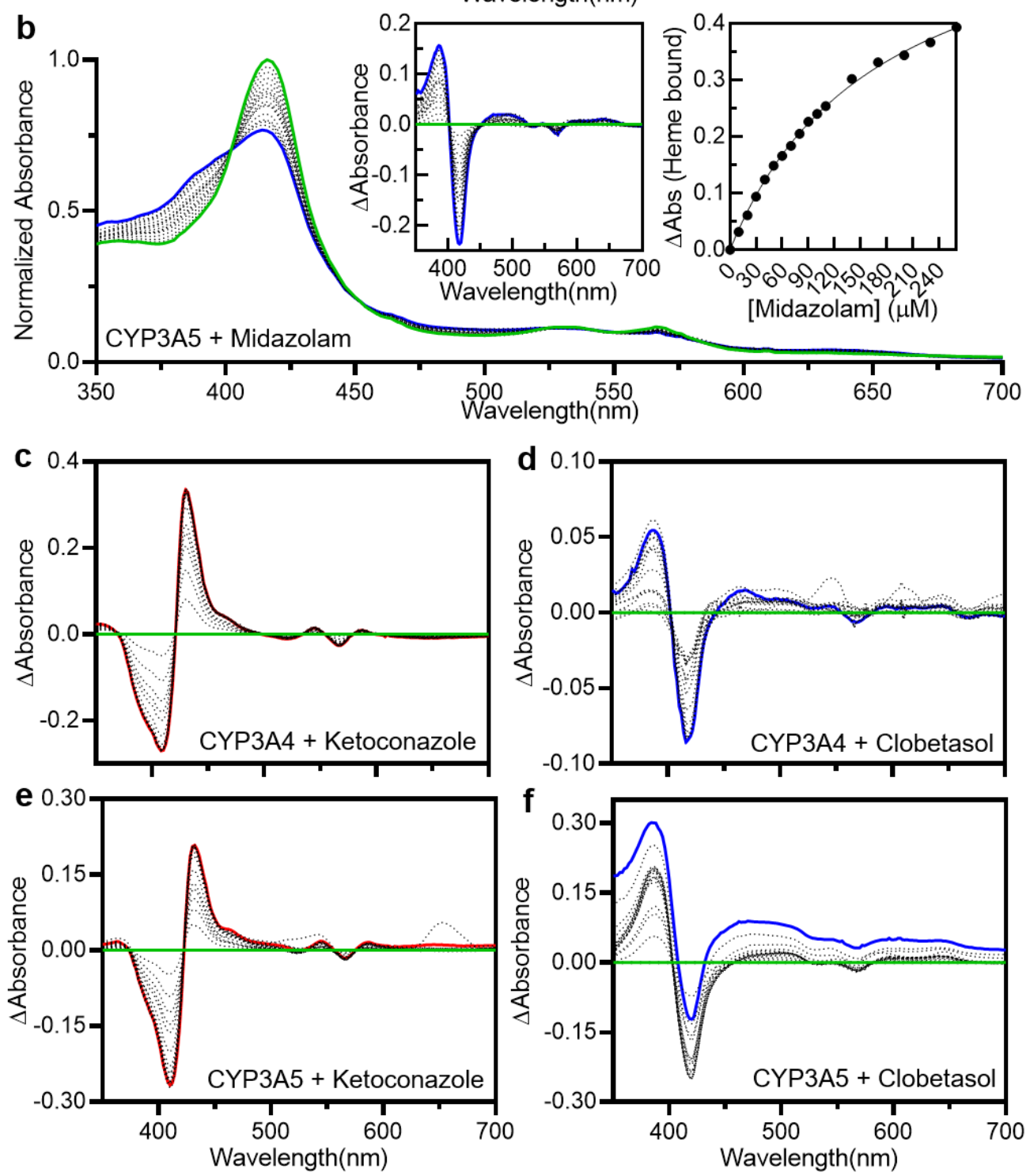


\section{Supplementary Figure 7. Spectral changes of CYP3A4 and CYP3A5 induced by various}

compounds. (a,b) Spectral changes upon titrating midazolam into $5 \mu \mathrm{M} \mathrm{CYP3A4}$ (a) and CYP3A5 (b). Absorbance values were normalized to the maximum value in the spectra. Left insets: Difference spectra showing absorbance changes between the ligand-free and midazolambound states. Right insets: Titration plots fitted to a hyperbola equation. (c-f) Difference spectra showing absorbance changes between the ligand-free and compound-bound states for CYP3A4 and CYP3A5 proteins. Color scheme: The UV-Vis spectrum for the ligand-free (resting) state is in green, whereas the spectra for the last titration point are in blue (type II blue shift) or red (type I red shift). 


\section{Supplementary Data}

Supplementary Data 1. Interactive results of the CYP3A5 inhibition screen. An interactive plot of the screening results from the $5 \mu \mathrm{M}$ compound screen for CYP3A5 inhibition. The file (Supplementary_Data1.html) can be downloaded from the following link:

https://osf.io/k273i/?view only=654c4d5c733642ad824535419095054f. Hover over a data point to see its name, \% CYP3A5 inhibition, and final concentration. Click and drag to zoom in.

\section{Supplementary Data 2. CYP3A5 expression across all cancer cohorts of The Cancer}

Genome Atlas (TCGA). Normalized expression of CYP3A5 in units of $\log 2$ (normalized $\mathrm{CPM}+1$ ) with corresponding cancer cohort abbreviations and their respective cancer types. 


\section{Supplementary Videos}

Supplementary Video 1. Molecular dynamics simulations of clobetasol-bound CYP3A4.

Full, 200-nanosecond simulations of clobetasol-bound CYP3A4 (Supplementary_Video1) can be viewed or downloaded at the following link:

https://osf.io/k273j/?view only=654c4d5c733642ad824535419095054f

Supplementary Video 2. Molecular dynamics simulations of clobetasol-bound CYP3A5.

Full, 200-nanosecond simulations of clobetasol-bound CYP3A5 (Supplementary_Video2) can be viewed or downloaded at the following link:

https://osf.io/k273j/?view only=654c4d5c733642ad824535419095054f 